\title{
Effects of glutamine on cytokines 1L-1 and TNF- $\alpha$ in rehabilitation and prognosis of patients with lobectomy
}

\author{
XUEFENG WANG ${ }^{*}$, LEI HUANG ${ }^{*}$, YANXIA QU, HONGMEI LV and XIAOHUA HE \\ Department of Rehabilitation, Daqing Oilfield General Hospital, Daqing, Heilongjiang 163001, P.R. China
}

Received August 28, 2017; Accepted June 18, 2018

DOI: $10.3892 /$ etm.2018.6443

\begin{abstract}
This study was designed to investigate the effects of glutamine on cytokines $1 \mathrm{~L}-1, \mathrm{TNF}-\alpha$ and prognosis of patients with lobectomy in the process of postoperative rehabilitation. A total of 78 patients with lung cancer who underwent lobectomy from January 2015 to January 2017 were selected in Daqing Oilfield General Hospital (Daqing, China). Patients were randomly divided into two groups, 39 patients in each group. Patients in the control group were treated with conventional treatment, while patients in the observation group were treated with both conventional and glutamine treatment. The levels of TNF- $\alpha$, endotoxin, serum IL-1, IL-10, IL-15, IL-18 and intercellular adhesion molecule-1 (ICAM-1), myeloperoxidase (MPO) activity, incidence of nausea and vomiting, pulmonary histopathological changes, prognosis, and rehabilitation (time in bed, hospital stay and lung function) were compared between the two groups. Within 1 year after treatment, most patients survived, except 2 patients in the observation group and 3 patients in the control group who died. The rate of postoperation infection in the observation group was slightly lower than that in the control group. After treatment, the levels of endotoxin and TNF- $\alpha$ in the observation group were significantly lower than those in the control group $(\mathrm{p}<0.05)$. After treatment, the serum levels of IL-1 and IL-10 were significantly higher and the serum levels of IL-15 and IL-18 were significantly lower in the observation group than those in the control group $(\mathrm{p}<0.05)$. The expression levels of ICAM-1 and MPO activity were significantly higher in the observation group than those in the control group $(p<0.05)$. No significant difference in the incidence of nausea and vomiting was found between the two groups $(p>0.05)$. The average postoperative bed rest and hospital stay
\end{abstract}

Correspondence to: Dr Xiaohua He, Department of Rehabilitation, Daqing Oilfield General Hospital, 9 Zhongkang Street, Saertu, Daqing, Heilongjiang 163001, P.R. China

E-mail: he_xiaohua9@163.com

*Contributed equally

Key words: glutamine, lobectomy, cytokines, rehabilitation, prognosis in the observation group were significantly shorter than those in the control group $(\mathrm{p}<0.05)$. The levels of forced expiratory volume in $1 \mathrm{sec}$ (FEV1), forced vital capacity (FVC) and peak expiratory flow rate (PEFR) in the observation group were significantly higher than those in the control group $(\mathrm{p}<0.05)$. The results indicated that glutamine treatment is effective in the postoperative rehabilitation of patients undergoing lobectomy. Glutamine can regulate the levels of IL-1 and TNF- $\alpha$, improve lung function, shorten bed rest and hospitalization days, promote patient's postoperative rehabilitation process, and improve patient's quality of life.

\section{Introduction}

Lung cancer is a common malignancy with a high mortality rate and increasing incidence in young people (1). In the last 50 years, many countries have reported that the incidence and mortality of lung cancer has significantly increased. The morbidity and mortality of lung cancer in men rank first among all malignant tumors, and second in women. The causes of lung cancer include smoking, air pollution, ionizing radiation, chronic lung infections and occupational and environmental exposure. A large number of data have shown that long-term smoking has been closely related to lung cancer. The incidence of lung cancer among urban residents has been higher than that in rural areas, which may be related to the presence of carcinogens in urban air pollution and smoke. Therefore, non-smoking should be advocated and urban environmental hygiene should be improved. Studies have demonstrated that long-term smokers were more likely to develop lung cancer than non-smokers, and the younger they started smoking, the more likely they were to develop lung cancer. In addition, smoking not only directly effected health, but also had a negative effect on the health of the people around them, which led to the increase of the prevalence rate of lung cancer in passive smokers $(2,3)$.

Clinical manifestations of lung cancer are complex. Tumor site, tumor complications, metastasis and pathological type can determine the severity of lung cancer. However, manifestations of early lung cancer mainly include cough, chest pain and hoarseness, but no typical clinical symptoms were observed. Therefore, the misdiagnosis rate is high and most patients were diagnosed at an advanced stage, which in turn delays the treatment $(4,5)$. The symptoms of central lung cancer appear early and are severe, and the symptoms of peripheral lung cancer 
appear late and are mild, even asymptomatic, so it is often found in physical examination. The symptoms of lung cancer contain local symptoms, systemic symptoms, extrapulmonary symptoms, infiltration and metastasis symptoms (6-9).

Lobectomy, as the main treatment of lung cancer, can improve patients' condition. However, the complication of lobectomy, such as postoperative pulmonary infection and acute respiratory distress, can seriously affect patients' lung function, which in turn reduces the treatment effect (10). Studies have shown that glutamine can promote nitrogen balance, inhibit the translocation of enterotoxin and bacterial into blood, maintain intestinal mucosal integrity, and promote systemic immune and intestinal immune function. In this study, 78 patients who underwent lobectomy for lung cancer were selected to investigate the effects of glutamine on cytokines, including IL-1 and $\mathrm{TNF}-\alpha$, and the prognosis of patients.

\section{Patients and methods}

General information. A total of 78 lung cancer patients who underwent lobectomy were randomly selected from January 2015 to January 2017 in Daqing Oilfield General Hospital. Patients were randomly divided into two groups, 39 cases in each group. Patients in control group included 21 males and 18 females, the age ranged from 43 to 76 years with an average age of $59.56 \pm 7.89$ years, body mass index (BMI) ranged from 19 to $25 \mathrm{~kg} / \mathrm{m}^{2}$ with an average value of $22.19 \pm 2.13 \mathrm{~kg} / \mathrm{m}^{2}$, ASA classification: 23 cases of grade I and 16 cases of grade II. Patients in the observation group included 23 males and 16 females, the age ranged from 43 to 78 years with an average value of $59.63 \pm 7.93$ years, and BMI ranged from 19 to $26 \mathrm{~kg} / \mathrm{m}^{2}$ with an average value of $22.21 \pm 2.19 \mathrm{~kg} / \mathrm{m}^{2}$, ASA classification: 22 cases of grade I and 17 cases of grade II. There were no significant differences in the basic information (such as sex and age) between the two groups $(\mathrm{p}>0.05)$ (Table I). This study was approved by the Ethics Committee of Daqing Oilfield General Hospital (Daqing, China). Signed informed consents were obtained from the patients or the guardians.

\section{Criteria}

Inclusion criteria (7). i) patients diagnosed with lung cancer who underwent lobectomy; ii) aged between 40 and 65 years; and iii) with ASA grade of I-II.

Exclusion criteria (11). i) patients combined with severe hypertension and cardiovascular and cerebrovascular diseases; ii) patients who cannot tolerate the drugs used in this study; iii) patients who received radiotherapy or immunosuppressive therapy; and iv) patients with severe gastrointestinal ulcers, blood system diseases and liver and kidney dysfunction.

Methods. The patients in both groups were treated with lobectomy. After operation, patients in the control group were treated with conventional methods including nutritional support, fluid resuscitation, anti-inflammatory treatment, fasting, gastrointestinal decompression, bed rest and other comprehensive treatments. Besides conventional treatment, intravenous infusion of $100 \mathrm{ml}$ of glutamine (SFDA approval no. H20153121; Hangzhou Minsheng Pharmaceutical Group Co., Ltd., Hangzhou, China) was performed twice a day in the observation group. Treatment was performed for 7 days for both groups.
Evaluation indicators. i) levels of TNF- $\alpha$ and endotoxin (12): peripheral venous blood $(5 \mathrm{ml})$ was extracted from each patient before and at 7 days after treatment, after centrifugation at $2,500 \mathrm{x}$ g for $5 \mathrm{~min}$ at $4^{\circ} \mathrm{C}$, the supernatant was collected and stored at $-20^{\circ} \mathrm{C}$ in a refrigerator; levels of TNF- $\alpha$ were measured by enzyme-linked immunosorbent assay and levels of endotoxin were measured using BET-24A bacterial endotoxin analyzer, the kit were provided by Beijing Weitonglihua Experimental Animal Technical Co., Ltd. (Beijing, China), all operations were performed in strict accordance to the manufacturer's instructions. ii) Levels of serum IL-1, IL-10, IL-15 and IL-18 (13): levels of IL-1, IL-10, IL-15 and IL-18 were detected by enzyme-linked immunosorbent assay. iii) Intercellular adhesion molecule-1 (ICAM-1) expression and myeloperoxidase (MPO) activity: after treatment, expression of ICAM-1 in serum was measured by immunohistochemistry, and MPO activity in 5\% lung tissue homogenate was measured by colorimetric method, all operations were performed in strict accordance with the manufacturer's instructions. iv) The occurrence of nausea and vomiting (14). v) Lung function indicators: the forced expiratory volume in $1 \mathrm{sec}$ (FEV1), forced vital capacity (FVC) and peak expiratory flow rate (PEFR). vi) Lung histopathological changes (15): lung tissue was fixed in 10\% formaldehyde solution, followed by H\&E staining. Lung histopathological changes were observed under microscopy (BX-42; Olympus,Tokyo, Japan).

Statistical analysis. SPSS 22.0 software (IBM Corp., Armonk, NY, USA) was used to analyze all data in this study. Measurement data were expressed by mean \pm standard deviation (SD), and processed by t-test. Count data were expressed by the number of cases and percentages, and processed by $\chi^{2}$ test. $\mathrm{P}<0.05$ was considered to indicate a statistically significant difference.

\section{Results}

Levels of endotoxin and TNF- $\alpha$. Before treatment, no significant differences in endotoxin and TNF- $\alpha$ were found between the two groups ( $\mathrm{p}>0.05)$. After treatment, the levels of endotoxin and TNF- $\alpha$ were significantly lower in observation group than those in control group $(\mathrm{p}<0.05)$ (Table II).

Levels of $I L-1, I L-10, I L-15$ and $I L-18$. Before treatment, no significant differences in the levels of IL-1, IL-10, IL-15 and IL-18 were found between the two groups ( $\mathrm{p}>0.05)$; after treatment, the levels of IL-1 and IL-10 were significantly higher and the levels of IL-15 and IL-18 were significantly lower in the observation group than those in the control group $(\mathrm{p}<0.05)$ (Table III).

ICAM-1 expression and MPO activity. Compared with the control group, the expression level of ICAM-1 and MPO activity in the observation group were significantly higher than those in the control group $(\mathrm{p}<0.05)$ (Table IV).

Survival, postoperational infection and adverse reactions. All patients survived in the observation and control groups 1 year after the surgery, except for 2 patients in the observation group ( 1 for heart attack and 1 for cerebral infarction) 
Table I. Comparison of basic information between two groups.

\begin{tabular}{lccccc}
\hline & & & \multicolumn{2}{c}{ ASA classification } \\
\cline { 5 - 6 } Groups & Male/female & Age (years) & BMI $\left(\mathrm{kg} / \mathrm{m}^{2}\right)$ & I & II \\
\hline Control $(\mathrm{n}=39)$ & $21 / 18$ & $59.56 \pm 7.89$ & $22.19 \pm 2.13$ & 23 & 16 \\
Observation $(\mathrm{n}=39)$ & $23 / 16$ & $59.63 \pm 7.93$ & $22.21 \pm 2.19$ & 22 & 17 \\
$\mathrm{t} / \chi^{2}$ & 0.044 & 0.029 & 0.084 & 0.130 & $>0.05$ \\
P-value & $>0.05$ & $>0.05$ & $>0.05$ & & \\
\hline
\end{tabular}

BMI, body mass index.

Table II. Comparison of endotoxin and TNF- $\alpha$ between two groups (mean $\pm(\mathrm{SD}), \mathrm{ng} / \mathrm{l})$.

\begin{tabular}{lccc}
\hline Time-points & Groups & Endotoxin & TNF- $\alpha$ \\
\hline Before treatment & Control $(\mathrm{n}=39)$ & $1.13 \pm 0.26$ & $78.23 \pm 18.71$ \\
& Observation $(\mathrm{n}=39)$ & $1.08 \pm 0.31$ & $76.29 \pm 19.03$ \\
& $\mathrm{t}$ value & 0.772 & 0.454 \\
& P-value & $>0.05$ & $>0.05$ \\
After treatment & Control (n=39) & $0.47 \pm 0.23$ & $49.78 \pm 13.54$ \\
& Observation (n=39) & $0.21 \pm 0.15$ & $30.16 \pm 10.27$ \\
& $\mathrm{t}$ value & 5.913 & 7.210 \\
& P-value & $<0.05$ & $<0.05$ \\
\hline
\end{tabular}

and 3 patients in the control group ( 2 for diabetes mellitus and 1 for heart attack). The survival between the observation and control groups was not significantly different, $p>0.05$. Forty infections were observed in the observation group, including 5 cases of mycoplasma, 4 cases of Streptococcus pneumoniae, 2 cases of Haemophilus influenzae, 1 case of influenza virus and 2 cases of unknown infection. Nine infections were observed in the control group, including 3 cases of mycoplasma, 3 cases of Streptococcus pneumoniae, 2 cases of influenza virus and 1 case of unknown infection. The rate of postoperational infection in the observation group was slightly lower than that in the control group. Four cases $(10.26 \%)$ of nausea and vomiting were observed in the observation group and 3 cases $(7.69 \%)$ were found in the control group, significant differences were found between the two groups $\left(\chi^{2}=0.000, \mathrm{p}>0.05\right)$.

Postoperative bed rest and hospital stay. Postoperative bed rest and hospital stay were significantly shorter in the observation group than those in the control group $(\mathrm{p}<0.05)$ (Table V).

Lung function indicator. Compared with the control group, FEV1, FVC and PEFR were significantly improved in the observation group $(\mathrm{p}<0.05)$ (Table VI).

Lung histopathological changes. Normal lung tissue structure was observed in the control group although a small amount of inflammatory cell infiltration appeared, but edema was not found. Finally, inflammatory cell infiltration and alveolar hemorrhage were significantly improved in the observation group (Fig. 1).

\section{Discussion}

Overview of glutamine. Glutamine is a precursor material for glutathione (GSH), pyrimidine, purine and amino acids. It is also a major source of energy for immune, lymph and fibroblasts in the body. Glutamine provides not only ATP but also materials for the synthesis of proteins and nucleic acids $(16,17)$. Some scholars believe that glutamine can play a two-way immune regulation role. It can inhibit the expression of inflammatory factors, but also improve immune function, and the protective role of glutamine is different in different organs (18). At present, the pharmacological effects of glutamine are mainly reflected in the following points: i) glutamine can promote protein synthesis, inhibit excessive muscle tissue decomposition, and prevent multiple organ dysfunction $(19,20)$; ii) glutamine can be converted to glutamate, and further promote liver synthesis of GSH, which is the most important antioxidant in the body, thereby enhancing the body's antioxidant capacity (21); iii) glutamine can participate in the energy and material metabolism of immune cells to reduce infection (22); iv) glutamine can reduce intestinal damage, prevent endotoxin and bacteria migration, protect the mucosal barrier, and reduce the risk of intestinal infection (17,23); and v) glutamine can reduce protein decomposition under stress conditions, reduce complications, and improve the overall condition (24).

Glutamine, TNF- $\alpha$ and endotoxin. Lobectomy, as the main treatment of advanced lung disease, can effectively remove the tumor lesions. However, systemic inflammatory response and other serious complications caused by lobectomy can seriously aggravate the patients' condition (25). Therefore, the identification of an effective measure to reduce the occurrence of multiple complications after surgery as well as improving the prognosis of patients is particularly important. TNF- $\alpha$ can directly kill human tumor cells, but has no significant toxicity to normal cells. As the most active factor in the process of killing tumor cells, TNF- $\alpha$ can reflect the conditions of patients (26). Endotoxin is composed of a non-specific core polysaccharide, cell-specific polysaccharide and lipid A. In this study, endotoxin and TNF- $\alpha$ levels in the observation group were significantly improved compared with those in 

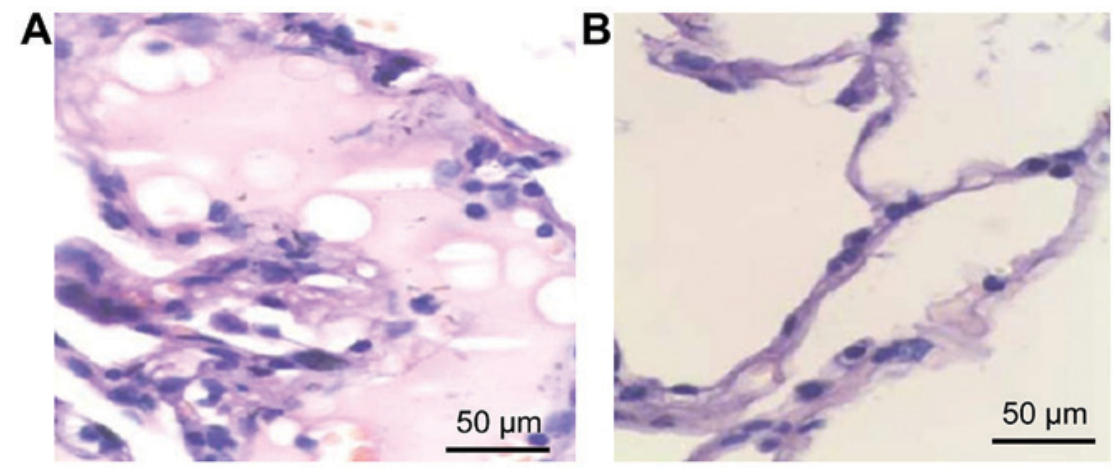

Figure 1. Lung histopathological changes. (A) Control group: Normal lung tissue structure, a small amount of inflammatory cell infiltration appeared and no edema was observed. (B) Observation group: Inflammatory cell infiltration and alveolar hemorrhage were significantly improved.

Table III. Comparison of serum factors between the two groups (mean $\pm(\mathrm{SD}), \mathrm{pg} / \mathrm{ml}$ ).

\begin{tabular}{lccccc}
\hline Time-points & Groups & IL-1 & IL-10 & IL-15 & IL-18 \\
\hline Before treatment & Control $(\mathrm{n}=39)$ & $27.23 \pm 10.97$ & $56.48 \pm 16.78$ & $51.78 \pm 12.31$ & $253.08 \pm 33.92$ \\
& Observation $(\mathrm{n}=39)$ & $26.86 \pm 11.34$ & $55.71 \pm 17.03$ & $52.01 \pm 12.09$ & $251.83 \pm 30.54$ \\
& $\mathrm{t}$ value & 0.147 & 0.201 & 0.083 & 0.171 \\
& P-value & $>0.05$ & $>0.05$ & $>0.05$ & $>0.05$ \\
After treatment & Control (n=39) & $31.04 \pm 17.69$ & $103.76 \pm 19.78$ & $29.17 \pm 7.82$ & $172.58 \pm 20.59$ \\
& Observation (n=39) & $39.78 \pm 18.37$ & $173.27 \pm 21.03$ & $10.91 \pm 6.34$ & $110.37 \pm 15.76$ \\
& t value & 2.140 & 15.036 & 11.327 & 14.983 \\
& P-value & $<0.05$ & $<0.05$ & $<0.05$ & $<0.05$ \\
\hline
\end{tabular}

Table IV. Comparison of the expression level of ICAM-1 and MPO activity between groups (mean $\pm(\mathrm{SD})$.

\begin{tabular}{lcc}
\hline Groups & ICAM-1 $(\%)$ & MPO (U/g) \\
\hline Control $(\mathrm{n}=39)$ & $85.71 \pm 10.34$ & $4.24 \pm 0.61$ \\
Observation $(\mathrm{n}=39)$ & $46.03 \pm 10.67$ & $13.95 \pm 1.92$ \\
t value & 16.678 & 30.100 \\
P-value & $<0.05$ & $<0.05$ \\
\hline
\end{tabular}

ICAM-1, intercellular adhesion molecule-1; MPO, myeloperoxidase.

Table V. Comparison of postoperative bed rest and hospital stay between the two groups.

\begin{tabular}{lcc}
\hline Groups & Bed rest & Hospital stay \\
\hline Control $(\mathrm{n}=39)$ & $2.7 \pm 0.7$ & $9.4 \pm 4.3$ \\
Observation $(\mathrm{n}=39)$ & $1.2 \pm 0.4$ & $6.2 \pm 2.8$ \\
t value & 14.412 & 4.831 \\
P-value & $<0.05$ & $<0.05$ \\
\hline
\end{tabular}

the control group, indicating that glutamine can reduce the postoperative inflammatory response and side-effects. This result is consistent with the finding of Cetinbas et al (27). Glutamine is the most abundant amino acid in cells and
Table VI. Comparison of lung function indicators between the two groups mean $\pm(\mathrm{SD})$.

\begin{tabular}{lccc}
\hline Groups & FEV1 (l) & FVC (l) & PEFR $(1 / \mathrm{sec})$ \\
\hline Control $(\mathrm{n}=39)$ & $1.26 \pm 0.24$ & $1.41 \pm 0.63$ & $2.34 \pm 1.33$ \\
Observation $(\mathrm{n}=39)$ & $1.79 \pm 0.32$ & $2.21 \pm 0.77$ & $3.38 \pm 1.52$ \\
t value & 3.928 & 2.538 & 2.084 \\
P-value & $<0.05$ & $<0.05$ & $<0.05$ \\
\hline
\end{tabular}

FEV1, forced expiratory volume in $1 \mathrm{sec}$; FVC, forced vital capacity; PEFR, peak expiratory flow rate.

human body. Under normal circumstances, glutamine has the characteristics of non-essential amino acids. Glutamine, which is important for the catabolic acceleration process, can improve metabolism of the intestinal mucosal cells; glutamine can promote the rapid synthesis of GSH substances, and further strengthen the body's antioxidant capacity (27). At the same time, as raw materials and nitrogen donor of nucleotide precursor, glutamine can greatly promote the differentiation and proliferation of macrophages and lymphocyte, increase the synthesis of phospholipid mRNA, and inhibit TNF- $\alpha$ over-production, and thus effectively improve immune function. In addition, glutamine can protect the mucosal barrier, prevent endotoxin and bacterial migration, reduce endotoxin levels and tissue damage (28). 
Glutamine, IL-1, IL-10, IL-15, IL-18, MPO and ICAM-1. IL-1 can promote the secretion of IL-2 substances from T cells by stimulating platelet growth factor to repair the body immune response system, thus effectively improve patients' condition. As a neutrophil-specific reductase, MPO level is stable in cells and MPO can be used to reflect neutrophil aggregation and activity. As a surface protein of endothelial cells, ICAM-1 can promote inflammatory response, control tumor tissue deterioration and metastasis, so as to regulate immune response. The expression level of ICAM-1 is low under normal conditions, and the increased level of ICAM-1 can lead to the binding of ICAM-1 to its specific receptor, which in turn enhances the adhesion of white blood cells, inflammatory cells and tumor cells to endothelial cells, so as to promote endothelial cell activation, resulting in excessive inflammatory response. In this study, the levels of IL-1 and IL-10, the expression level of ICAM-1 and MPO activity were significantly higher, and the levels of IL-15 and IL-18 were significantly lower in the observation group than those in the control group, indicating that glutamine could effectively reduce the inflammatory response, and improve body immunity. The possible explanation is that, as an important antioxidant, glutamine can increase the level of GSH under stress conditions, reduce endothelial cell damage, stabilize cell membrane and protein structure, promote antioxidant capacity, and protect cell function. At the same time, glutamine can enhance immune function, promote cell metabolism, prevent muscle over-decomposition, and reduce damage to tissues and organs. Glutamine can also enhance the immune function, reduce the production of inflammatory factors, and effectively regulate the levels of cytokines (29).

Glutamine and prognosis. In this study, no significant differences in the incidence of nausea and vomiting were found between the two groups. But observation under microscope revealed that postoperative recovery of the observation group was significantly better than that of the control group, indicating that glutamine plays an important role in energy metabolism and immunity. In addition, average postoperative bed rest and hospital stay in the observation group were significantly shorter than those in the control group ( $\mathrm{p}<0.05)$. FEV1, FVC and PEFR in the observation group were also significantly better than those in the control group $(\mathrm{p}<0.05)$, indicating that glutamine can promote the recovery of lung cancer patients after lobectomy.

In conclusion, glutamine can regulate the levels of cytokines IL-1, IL-10, IL-15 and IL-18 in patients with lobectomy to improve immune function and promote postoperative rehabilitation.

\section{Acknowledgements}

Not applicable.

\section{Funding}

No funding was received.

\section{Availability of data and materials}

The datasets used and/or analyzed during the present study are available from the corresponding author on reasonable request.

\section{Authors' contributions}

XW, LH and XH conceived and designed the study. XW, YQ and HL were responsible for the collection and analysis of the patient data. LH and XH interpreted the data and drafted the manuscript. XW revised the manuscript critically for important intellectual content. All authors have read and approved the final manuscript.

\section{Ethics approval and consent to participate}

The study was approved by the Ethics Committee of Daqing Oilfield General Hospital (Daqing, China). Signed informed consents were obtained from the patients or the guardians.

\section{Patient consent for publication}

Not applicable.

\section{Competing interests}

The authors declare that they have no competing interests.

\section{References}

1. Vlahos I: Dilemmas in lung cancer staging. Radiol Clin North Am 56: 419-435, 2018.

2. Wang Y, Jiang M, Du C, Yu Y, Liu Y, Li M and Luo F: Utilization of lung cancer cell lines for the study of lung cancer stem cells. Oncol Lett 15: 6791-6798, 2018.

3. de Sousa VML and Carvalho L: Heterogeneity in lung cancer. Pathobiology 85: 96-107, 2018.

4. Sharma A and Shepard JO: Lung cancer biopsies. Radiol Clin North Am 56: 377-390, 2018.

5. Shroff GS, Viswanathan C, Carter BW, Benveniste MF, Truong MT and Sabloff BS: Staging lung cancer: Metastasis. Radiol Clin North Am 56: 411-418, 2018.

6. Brand $\mathrm{T}$ and Haithcock B: Lung cancer and lung transplantation. Thorac Surg Clin 28: 15-18, 2018.

7. Hayashi Y, Sawa Y, Fukuyama N, Nakazawa H and Matsuda H: Preoperative glutamine administration induces heat-shock protein 70 expression and attenuates cardiopulmonary bypass-induced inflammatory response by regulating nitric oxide synthase activity. Circulation 106: 2601-2607, 2002.

8. Fahrner R, Trochsler M, Corazza N, Graubardt N, Keogh A, Candinas D, Brunner T, Stroka D and Beldi G: Tumor necrosis factor-related apoptosis-inducing ligand on NK cells protects from hepatic ischemia-reperfusion injury. Transplantation 97: 1102-1109, 2014.

9. Souba WW, Klimberg VS, Plumley DA, Salloum RM, Flynn TC, Bland KI and Copeland EM III: The role of glutamine in maintaining a healthy gut and supporting the metabolic response to injury and infection. J Surg Res 48: 383-391, 1990.

10. Zou XP, Chen M, Wei W, Cao J, Chen L and Tian M: Effects of enteral immunonutrition on the maintenance of gut barrier function and immune function in pigs with severe acute pancreatitis. JPEN J Parenter Enteral Nutr 34: 554-566, 2010.

11. Peng ZY, Hamiel CR, Banerjee A, Wischmeyer PE, Friese RS and Wischmeyer P: Glutamine attenuation of cell death and inducible nitric oxide synthase expression following inflammatory cytokine-induced injury is dependent on heat shock factor-1 expression. JPEN J Parenter Enteral Nutr 30: 400-406; discussion 406-7, 2006.

12. Von Bültzingslöwen I, Adlerberth I, Wold AE, Dahlén G and Jontell M: Oral and intestinal microflora in 5-fluorouracil treated rats, translocation to cervical and mesenteric lymph nodes and effects of probiotic bacteria. Oral Microbiol Immunol 18: 278-284, 2003.

13. Singleton KD, Serkova N, Banerjee A, Meng X, Gamboni-Robertson F and Wischmeyer PE: Glutamine attenuates endotoxin-induced lung metabolic dysfunction: Potential role of enhanced heat shock protein 70. Nutrition 21: 214-223, 2005. 
14. Butler EB,Zhao Y, Muñoz-Pinedo C,Lu J and Tan M: Stalling the engine of resistance: Targeting cancer metabolism to overcome therapeutic resistance. Cancer Res 73: 2709-2717, 2013.

15. Barquissau V, Beuzelin D, Pisani DF, Beranger GE, Mairal A, Montagner A, Roussel B, Tavernier G, Marques MA, Moro C, et al: White-to-brite conversion in human adipocytes promotes metabolic reprogramming towards fatty acid anabolic and catabolic pathways. Mol Metab 5: 352-365, 2016

16. Kou Y, Zheng WT and Zhang YR: Inhibition of miR-23 protects myocardial function from ischemia-reperfusion injury through restoration of glutamine metabolism. Eur Rev Med Pharmacol Sci 20: 4286-4293, 2016.

17. Tanaka K, Sasayama T, Irino Y, Takata K, Nagashima H, Satoh N, Kyotani K, Mizowaki T, Imahori T, Ejima Y, et al Compensatory glutamine metabolism promotes glioblastoma resistance to mTOR inhibitor treatment. J Clin Invest 125: 1591-1602, 2015

18. Jacque N, Ronchetti AM, Larrue C, Meunier G, Birsen R, Willems L, Saland E, Decroocq J, Maciel TT, Lambert M, et al: Targeting glutaminolysis has antileukemic activity in acute myeloid leukemia and synergizes with BCL-2 inhibition. Blood 126: 1346-1356, 2015.

19. McRae MP: Therapeutic benefits of glutamine: An umbrella review of meta-analyses. Biomed Rep 6: 576-584, 2017.

20. Yuen CA, Asuthkar S, Guda MR, Tsung AJ and Velpula KK: Cancer stem cell molecular reprogramming of the Warburg effect in glioblastomas: A new target gleaned from an old concept. CNS Oncol 5: 101-108, 2016.

21. Pusapati RV, Daemen A, Wilson C, Sandoval W, Gao M, Haley B, Baudy AR, Hatzivassiliou G, Evangelista M and Settleman J: MTORC1-dependent metabolic reprogramming underlies escape from glycolysis addiction in cancer cells. Cancer Cell 29: $548-562,2016$

22. Csibi A, Lee G, Yoon SO, Tong H, Ilter D, Elia I, Fendt SM, Roberts TM and Blenis J: The mTORC1/S6K1 pathway regulates glutamine metabolism through the eIF4B-dependent control of c-Myc translation. Curr Biol 24: 2274-2280, 2014.
23. Kamata S, Kishimoto T, Kobayashi S, Miyazaki M and Ishikura H: Possible involvement of persistent activity of the mammalian target of rapamycin pathway in the cisplatin resistance of AFP-producing gastric cancer cells. Cancer Biol Ther 6: 1036-1043, 2007.

24. Gini B, Zanca C, Guo D, Matsutani T, Masui K, Ikegami S, Yang H, Nathanson D, Villa GR, Shackelford D, et al: The mTOR kinase inhibitors, CC214-1 and CC214-2, preferentially block the growth of EGFRvIII-activated glioblastomas. Clin Cancer Res 19: 5722-5732, 2013.

25. Pochini L, Scalise $M$, Galluccio $M$ and Indiveri $C$ : Membrane transporters for the special amino acid glutamine: Structure/function relationships and relevance to human health. Front Chem 2: 61, 2014

26. Liu W, Le A, Hancock C, Lane AN, Dang CV, Fan TW and Phang JM: Reprogramming of proline and glutamine metabolism contributes to the proliferative and metabolic responses regulated by oncogenic transcription factor c-MYC. Proc Natl Acad Sci USA 109: 8983-8988, 2012.

27. Cetinbas F, Yelken B and Gulbas Z: Role of glutamine administration on cellular immunity after total parenteral nutrition enriched with glutamine in patients with systemic inflammatory response syndrome. J Crit Care 25: 661.e1-661.e6, 2010.

28. Mousavi SN, Samini F, Nematy M, Philippou E, Safarian M, Tavallaiee $\mathrm{S}$ and Norouzy A: Hyperglycemia and antibody titres against heat shock protein 27 in traumatic brain injury patients on parenteral nutrition. Iran J Basic Med Sci 17: 119-122, 2014.

29. Chen H, Chen D, Michiels J and De Smet S: Dietary fiber affects intestinal mucosal barrier function by regulating intestinal bacteria in weaning piglets. Commun Agric Appl Biol Sci 78: 71-78, 2013.

This work is licensed under a Creative Commons Attribution-NonCommercial-NoDerivatives 4.0 International (CC BY-NC-ND 4.0) License. 